\title{
Correspondence
}

\section{Spider taxonomists catch data on web}

A successful systematics initiative in arachnology could provide an invaluable model for rapid delivery of taxonomic data for other animal groups. Until now, the inaccessibility of the classical and obscure taxonomic literature has been a major hindrance to the field's progress.

The World Spider Catalog (www.wsc.nmbe.ch), launched last year, now contains complete taxonomic data for almost 46,000 validated spider species and an embedded collection of 13,000 references. Spiders are the most species-rich terrestrial invertebrate group after insects.

More than $97 \%$ of the world's spider literature was collected within just 600 days of communicating our goal to the research community. The database logs a daily average of 600 hits and 400 downloads.

Wolfgang Nentwig University of Bern, Switzerland.

Daniel Gloor Natural History

Museum Bern, Switzerland.

Christian Kropf University

of Bern; and Natural History

Museum Bern, Switzerland.

wolfgang.nentwig@iee.unibe.ch

\section{Bury the idea that soils are a local issue}

As the International Year of Soils ends, we agree that the importance of integrating soils into policies to tackle global challenges cannot be underestimated (see L. Montanarella Nature 528, 32-33; 2015). Soils are not a local issue they 'move' at time and space scales that are relevant to global policy.

For example, Saharan soil dust has boosted Atlantic plankton (E. Marañón et al. Limnol. Oceanogr. 55, 2339-2352; 2010) and tree growth in Amazonian forests (R. Swap et al. Tellus B 44, 133-149; 1992). There are environmental consequences beyond national borders when pollutants and nutrients that are attached to soil particles

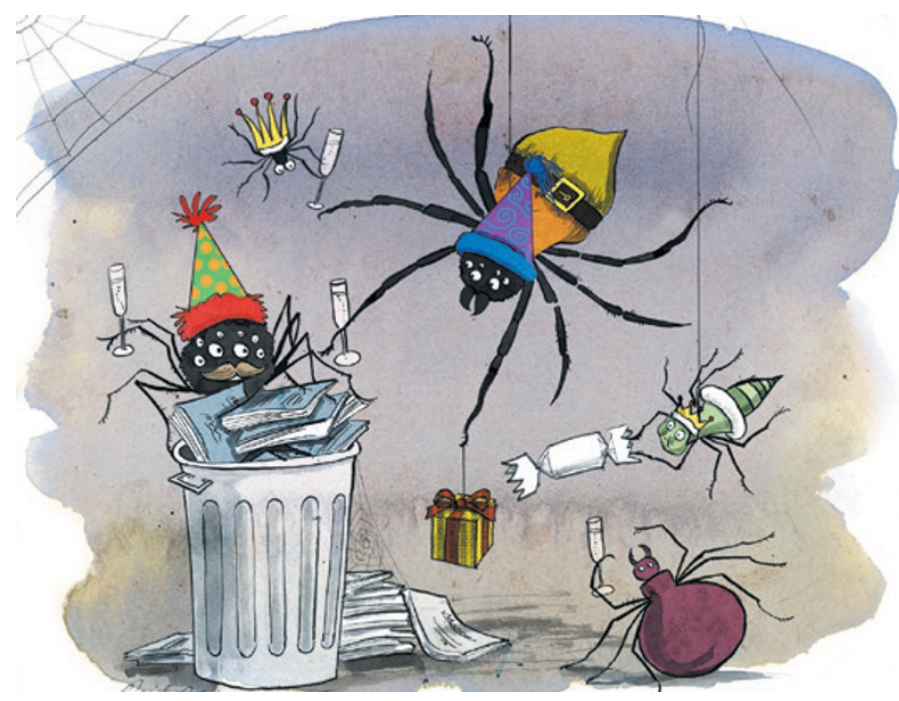

enter waterways, or when soil nitrates leach into aquifers. The influence of soil management on climate is also global because of its carbon-storage capacity and interactions with greenhouse gases. Changes in soil-surface reflection (albedo) affect energy balance, climate and weather. Frank G. A. Verheijen, Ana C. Bastos University of Aveiro, Portugal.

Simon Jeffery Harper Adams University, Newport, UK. frankverheijen@gmail.com

\section{Labs should cut plastic waste too}

Many governments now impose charges for single-use plastic bags and bottles. As responsible researchers, we should cut back on disposable plastics (see also G. Bistulfi Nature 502, 170; 2013).

We estimate that the 280 bench scientists in our bioscience department generated roughly 267 tonnes of plastic in 2014 (data from University of Exeter Sustainability and Waste and Resource Management offices). That is equivalent to about 5.7 million empty 2-litre plastic bottles. Some 20,500 institutions worldwide are involved in biological, medical or agricultural research (where plastic disposal is likely to be heaviest), so that could equate to around 5.5 million tonnes of lab plastic waste in 2014 roughly the combined tonnage of 67 cruise liners, and equal to $83 \%$ of the plastic recycled worldwide in 2012.

We justify our use of disposables on the grounds of costs and time saved. Grant agencies therefore need to introduce incentives to reduce plastic waste, for example by funding lab washing-up and recycling facilities, and possibly to make greener lab practices a requirement in the grantapplication process.

Mauricio A. Urbina University of Exeter, UK; and University of Concepción, Chile.

Andrew J. R.Watts, Erin E.

Reardon University of Exeter, UK. mauriciourbina@udec.cl

\section{Nuclear industry no model for biosafety}

I applaud Tim Trevan's call to reform lab biosafety, but disagree with his argument for using the nuclear industry as a model (Nature 527, 155-158; 2015).

Nuclear facilities are strictly regulated and ensure that potential hazards arising from process changes are engineered out (see go.nature.com/qyzoth). Yet scientists are not processdriven: being autonomous and creative, they need freedom to change and require a dynamic safety culture that can accommodate new challenges. These include the replacement of humans by technology, reduced supervision and declining safety competencies - none of which applies to the nuclear industry.

Chasing a 'zero harm' mantra can actually promote a poor safety culture because it is an outcome rather than a goal (for examples of alternative approaches, see go.nature.com/xgupio and go. nature.com/gcjqfl). As Trevan points out, an effective safety culture is measured through engagement, understanding and care for everyone's well-being. Chris Lea UCB Celltech, Slough, UK. chris.lea@ucb.com

\section{Bond villain fails neuroanatomy}

The thrills and action in Spectre, the latest James Bond film, were somewhat marred for this viewer by a fundamental neuroanatomical blunder.

The scene is a Moroccan desert. Bond's nemesis is torturing our hero using a head clamp fused with a robotic drill. Intending to erase Bond's memory of faces, the villain says he is directing his drill to the (lateral) "fusiform gyrus" correctly identifying a core brain area for facial recognition (J. Parvizi et al. J. Neurosci. 32, 14915-14920; 2012).

But the film-makers got it wrong. Whereas the drill should have been aimed just in front of 007's ear, it was directed below the mastoid process under and behind his left ear. There it would have met the lateral part of the first or second cervical vertebra, perhaps hitting the ipsilateral vertebral artery and triggering a stroke or massive haemorrhage. Unless fatal, it certainly would not have deleted the bank of faces in Bond's memory. Michael D. Cusimano St Michael's Hospital, University of Toronto, Canada. mountain@smh.ca 